\title{
Fluid entrainment by isolated vortex rings
}

\author{
By JOHN O. DABIRI AND MORTEZA GHARIB \\ Bioengineering \& Graduate Aeronautical Laboratories, California Institute of Technology, \\ Pasadena, CA 91125, USA
}

(Received 10 December 2003 and in revised form 15 April 2004)

Of particular importance to the development of models for isolated vortex ring dynamics in a real fluid is knowledge of ambient fluid entrainment by the ring. This time-dependent process dictates changes in the volume of fluid that must share impulse delivered by the vortex ring generator. Therefore fluid entrainment is also of immediate significance to the unsteady forces that arise due to the presence of vortex rings in starting flows. Applications ranging from industrial and transportation, to animal locomotion and cardiac flows, are currently being investigated to understand the dynamical role of the observed vortex ring structures. Despite this growing interest, fully empirical measurements of fluid entrainment by isolated vortex rings have remained elusive. The primary difficulties arise in defining the unsteady boundary of the ring, as well as an inability to maintain the vortex ring in the test section sufficiently long to facilitate measurements. We present a new technique for entrainment measurement that utilizes a coaxial counter-flow to retard translation of vortex rings generated from a piston-cylinder apparatus, so that their growth due to fluid entrainment can be observed. Instantaneous streamlines of the flow are used to determine the unsteady vortex ring boundary and compute ambient fluid entrainment. Measurements indicate that the entrainment process does not promote self-similar vortex ring growth, but instead consists of a rapid convection-based entrainment phase during ring formation, followed by a slower diffusive mechanism that entrains ambient fluid into the isolated vortex ring. Entrained fluid typically constitutes $30 \%$ to $40 \%$ of the total volume of fluid carried with the vortex ring. Various counter-flow protocols were used to substantially manipulate the diffusive entrainment process, producing rings with entrained fluid fractions up to $65 \%$. Measurements of vortex ring growth rate and vorticity distribution during diffusive entrainment are used to explain those observed effects, and a model is developed to relate the governing parameters of isolated vortex ring evolution. Measurement results are compared with previous studies of the process, and implications for the dynamics of starting flows are suggested.

\section{Introduction}

The earliest theoretical treatments of isolated vortex ring kinematics and dynamics predate quantitative experimental observations of the same phenomena by a halfcentury, due largely to the lack of available techniques to measure the salient fluid mechanics. Although ideal vortex ring models such as those of Saffman (1970), Fraenkel (1972), and Norbury (1973) have remained popular for describing empirical vortex ring dynamics (e.g. Gharib, Rambod \& Shariff 1998; Linden \& Turner 2001), the past few decades of research have witnessed several efforts to reconcile the predictions of analytical models with more recent observations made in the laboratory. 
The most common method for generating vortex rings in the laboratory is the piston-cylinder arrangement. In this configuration, a cylindrical piston with outer diameter flush to the inner diameter of a hollow cylinder is translated axially from rest. A fluid efflux emerges after separating from the sharp-edged lip at the open end of the hollow cylinder. The resulting cylindrical vortex sheet induces its own roll-up into a ring which propagates axially away from the exit plane of the generator.

Vortex rings generated in the laboratory tend to depart from the thin-ring limit typically studied in theory. Nevertheless the literature contains notable examples of agreement between classical theoretical predictions and empirical observations (e.g. Widnall \& Sullivan 1973; Liess \& Didden 1976).

Maxworthy (1972) credits Reynolds (1876) with the first qualitatively correct observations of vortex ring propagation subsequent to the formation process. Specifically, Reynolds describes the growth of the ring in time due to entrainment of fluid surrounding the ring. Assuming the ring itself possesses a nominally constant impulse, the ring velocity decreases as a consequence of shared momentum with an increasing mass of fluid.

A physical mechanism for ambient fluid entrainment consisting of repeated cycles of viscous diffusion and circulatory transport is proposed by Maxworthy (1972). Vorticity from the core of the isolated vortex ring diffuses to its radial extent, where it interacts with oncoming irrotational fluid (in the reference frame of the vortex ring). A process of viscous dissipation reduces the total pressure of the fluid adjacent to the ring, to the point that it cannot pass over the ring and is instead entrained by the circulatory motion of the ring. A portion of the diffused vorticity will return to the ring in this entrainment process, while the remainder will form a small wake behind the ring. Formation of the wake is facilitated by free-stream fluid that still possesses sufficient total pressure after the dissipation process to convect the vorticity downstream.

Using dimensional analysis and physical arguments regarding the structure of the dissipation region, Maxworthy (1972) derives the rate of fluid entrainment as

$$
\frac{\mathrm{d} \Omega_{B}}{\mathrm{~d} t}=C v^{1 / 2} a^{3 / 2} U_{v}^{1 / 2},
$$

where $\Omega_{B}$ is the volume of the vortex bubble (i.e. the volume of fluid moving with the ring), $v$ is the kinematic viscosity, $a$ is a characteristic dimension of the ring, and $U_{v}$ is the characteristic velocity of the free-stream fluid relative to the ring. The quantity $C$ is a dimensionless lumped parameter that incorporates the shape and size of the dissipation region, assuming self-similar vortex bubble growth. Equation (1.1) is used in concert with an assumption of constant vortex ring impulse to predict a $-2 / 3$ power-law decay of ring circulation in time, and to hypothesize the existence of a small wake of vorticity behind the ring.

Despite this progress, Maxworthy (1972) does not attempt to estimate the magnitude of the lumped parameter and therefore cannot attain a quantitative result for the contribution of entrainment to the dynamics of vortex rings. Maxworthy (1972) also lacks a method to empirically verify the predictions of ring circulation decay and wake formation. The validity of the assumption of constant ring impulse remains unknown, although Maxworthy (1972) limits it to high Reynolds numbers and small time scales.

Baird, Wairegi \& Loo (1977) briefly address the role of fluid entrainment quantitatively, using the classical slug model (cf. Shariff \& Leonard, 1992) to approximate the fraction of vortex ring fluid that originated in the free stream. They 
arrive at the result that $1 / 4$ of the fluid in the vortex ring is supplied by entrainment. The validity of the calculation is limited to small piston stroke length-to-diameter ratios - less than $2 L / D$ by their own assertion - so that it can be assumed that all of the impulse delivered from the vortex generator is transferred to the vortex ring. Confirmation of the prediction is not reported in those experiments or in subsequent work.

Müller \& Didden (1980) visualize the growth of vortex rings using a dye marker in the flow, and estimate the entrained fluid fraction of the vortex ring to be approximately $40 \%$. As this was an estimated average value, no conclusions regarding the temporal dependence of the process can be made.

Transient measurements of fluid entrainment in vortex rings are especially cumbersome due to difficulty in observing and defining the boundary of the vortex ring as it propagates in time. Maxworthy (1972) notes that the physical extent of the ring may not be accurately determined from common dye visualizations, due to marked differences in the diffusion coefficients of the vorticity and the dye marker (e.g. Schmidt number $\sim 10^{2}$ for Rhodamine WT). As such, he notes the propensity to misinterpret qualitative visualizations of the entrainment process (e.g. Prandtl \& Tietjens 1934). Fully quantitative measurements of unsteady ambient fluid entrainment by isolated vortex rings have not yet been accomplished.

The task of measuring fluid entrainment is simplified if the ring can be maintained within the measurement window for longer periods of time. In the absence of external intervention, the ring will propagate away from the vortex generator under its selfinduced velocity. The time allotted for an entrainment measurement will then be dictated by the length of the viewing window and the ring speed.

A set of experiments was conducted to demonstrate use of an axisymmetric counterflow to maintain the vortex ring within the measurement window for longer periods of time, with the goal of observing ring growth due to fluid entrainment. Instantaneous streamlines of the flow in the reference frame of the vortex ring were measured using quantitative flow visualization techniques. These data enabled definition of the unsteady boundary of the vortex ring for transient entrainment calculations.

Although convective transport dominates the fluid entrainment process during vortex ring formation, the focus of this paper is on the diffusive mechanism of ambient fluid entrainment that is observed in isolated vortex rings. Various counter-flow protocols were implemented to manipulate this entrainment process and to elucidate the governing physical principles. Based on these results, a model is developed to quantitatively relate the salient parameters of isolated vortex ring evolution. The experimental data obtained here are compared and contrasted with the work of Maxworthy (1972) and the estimates of Baird et al. (1977) and Müller \& Didden (1980). Finally, implications for the dynamics of starting flows are suggested.

\section{Measurement techniques}

\subsection{Apparatus}

Experiments were conducted in a $60 \mathrm{~cm} \mathrm{H} \times 40 \mathrm{~cm} \mathrm{~W} \times 110 \mathrm{~cm} \mathrm{~L}$ water tank using an impulsively started piston-cylinder arrangement similar to that described by Gharib et al. (1998). A basic schematic of the apparatus is shown in figure 1 (adapted from Krueger, Dabiri \& Gharib 2003). The piston is driven by an external water supply that is maintained at constant total head $(\Delta p=8.2 \mathrm{kPa})$ and delivered by a computercontrolled solenoid valve (ASCO Valve, Inc.), to provide a repeatable piston velocity profile. Fluid ejected from the open end of the sharp-edged hollow cylinder (inner 


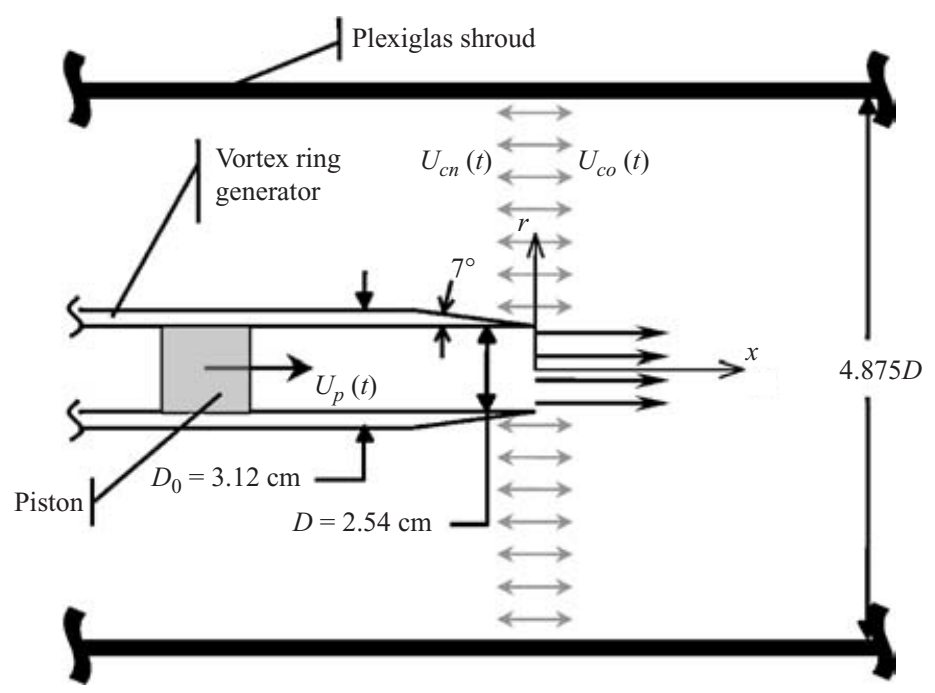

FigURE 1. Schematic of apparatus for piston-cylinder experiments (adapted from Krueger et al. 2003).

diameter $=2.54 \mathrm{~cm}$ ) rolls into a vortex ring and propagates through the surrounding fluid.

A modification to the traditional piston-cylinder arrangement was made by enclosing the primary cylinder in a $12 \mathrm{~cm}$ diameter coaxial shroud. An external pump (Leeson Electronic Corp.) connected to the shroud drives flow around the primary cylinder in either a co-flowing $(c o)$ or counter-flowing $(c n)$ configuration. Flow conditioning screens ensure nominally uniform flow around the primary cylinder. The shroud flow is also regulated by a computer-controlled solenoid valve. Tests verified that the presence of the shroud did not affect the observable vortex dynamics.

Ultrasonic flow probes (Transonic Systems, Inc.) measure the flow rates in the cylinder and shroud. All flow data are recorded to a computer hard disk via a LabVIEW (National Instruments) program. The starting jet flows are visualized quantitatively using digital particle image velocimetry (DPIV, cf. Willert \& Gharib 1991). The water tank is seeded with 13-micron (nominally) glass spheres that reflect light at the incident wavelength. Illumination is provided by a double-pulsed Nd:YAG laser (New Wave Research) that delivers $30 \mathrm{~mJ}$ of energy per pulse at $532 \mathrm{~nm}$. The laser beam is collimated by a cylindrical lens before entering the test section, which measures approximately $12 \mathrm{~cm}$ radially and $20 \mathrm{~cm}$ axially downstream of the vortex generator exit plane.

Mie scattering from the seeded water is captured by a $1024 \times 1024$ pixel blackand-white CCD digital camera (Uniq Vision, Inc.) at $30 \mathrm{~Hz}$. The pixel resolution corresponds to a physical test section resolution of approximately $0.19 \times 0.19 \mathrm{~mm}$. This is sufficient to resolve the vortex ring core vorticity distribution. Image data are transferred in real time by a progressive scan protocol to a frame grabber (Coreco Imaging) linked to a PC.

Images are paired according to the method described by Willert \& Gharib (1991). In the present case, each pair of images represents a separation of $18 \mathrm{~ms}$ between laser pulses. This timing results in an average particle shift of 4-7 pixels between images for the nominal piston speed of $5.5 \mathrm{~cm} \mathrm{~s}^{-1}$. Interrogation is accomplished using a window size of $32 \times 32$ pixels with a $50 \%$ overlap. Calculations of velocity and vorticity 
Notation Vortex generator $L / D$ Counter-flow speed Initiation delay

$\begin{array}{lccc}L D 2-C F 0 & 2 & 0 & \mathrm{~N} / \mathrm{A} \\ L D 2-C F 05-12 & 2 & 0.5 U_{p} & 12 L / D \\ L D 2-C F E & 2 & \left.U_{v}\right|_{C F 0} & 0 \\ L D 4-C F 0 & 4 & 0 & \mathrm{~N} / \mathrm{A} \\ L D 4-C F 05-2 & 4 & 0.5 U_{p} & 2 L / D \\ L D 4-C F 05-6 & 4 & 0.5 U_{p} & 6 L / D \\ L D 4-C F E & 4 & \left.U_{v}\right|_{C F 0} & 0\end{array}$

TABLE 1. Counter-flow protocols.

fields are completed using an in-house code on an Intel 2-GHz processor. Velocity and vorticity measurements possess an uncertainty of $1 \%$ and $3 \%$, respectively. A MATLAB (The Mathworks, Inc.) algorithm was created to rapidly visualize the instantaneous streamlines of the flow, based on the measured velocity field.

The flow Reynolds number is 1400 based on the piston speed and cylinder exit diameter, and varies between 2000 and 4000 based on the ring circulation (i.e. loop integral of measured velocity along a path enclosing the vortex).

\subsection{Counter-flow protocols}

A variety of counter-flow protocols were implemented to manipulate the dynamics of vortex rings generated by the piston-cylinder apparatus. The primary goal of these protocols was to maintain the vortex rings in the test section for sufficient time to permit transient fluid entrainment measurements after initial ring formation. In addition, several of the protocols were used to affect the vorticity distribution of the rings via the dynamics of the shear layer efflux from the vortex generator. The strength and convective velocity of the shear layer were affected by the counter-flow, increasing the former and decreasing the latter. These effects resulted in a modified vorticity distribution in the vortex ring. The dynamics of the entrainment process were significantly altered by changing the vorticity distribution in some cases, as will be shown in $\S 3$.

In the absence of counter-flow, vortex ring pinch-off (i.e. dynamic separation from the vortex generator flow source, cf. Gharib et al. 1998) was observed to occur at a formation number $F=3.6$, where

$$
F=\frac{\left.\overline{U_{p}} t\right|_{\text {pinch-off }}}{D}=\frac{\left.L\right|_{\text {pinch-off }}}{D} .
$$

In (2.1), $\overline{U_{p}}$ is the running average of the piston velocity, $L$ is the total piston stroke length (i.e. at the end of fluid ejection), $D$ is the cylinder exit diameter, and $t$ is time measured relative to the start of piston motion. Hence, counter-flow protocols initiated prior to $L / D=3.6$ affected the vortex ring vorticity distribution, whereas those initiated subsequent to pinch-off could not.

The magnitude of the counter-flow was assigned one of two values. In the first case it was matched to the measured vortex ring propagation speed $U_{v}$ in the absence of counter-flow. This enabled vortex rings to be maintained at a single axial location for longer periods of time. Alternatively, the counter-flow was set to one-half of the nominal piston speed. This is the theoretical vortex ring propagation speed predicted by the slug model (Baird et al. 1977). Table 1 lists the set of counter-flow protocols utilized in these experiments, along with an abbreviated notation to be 

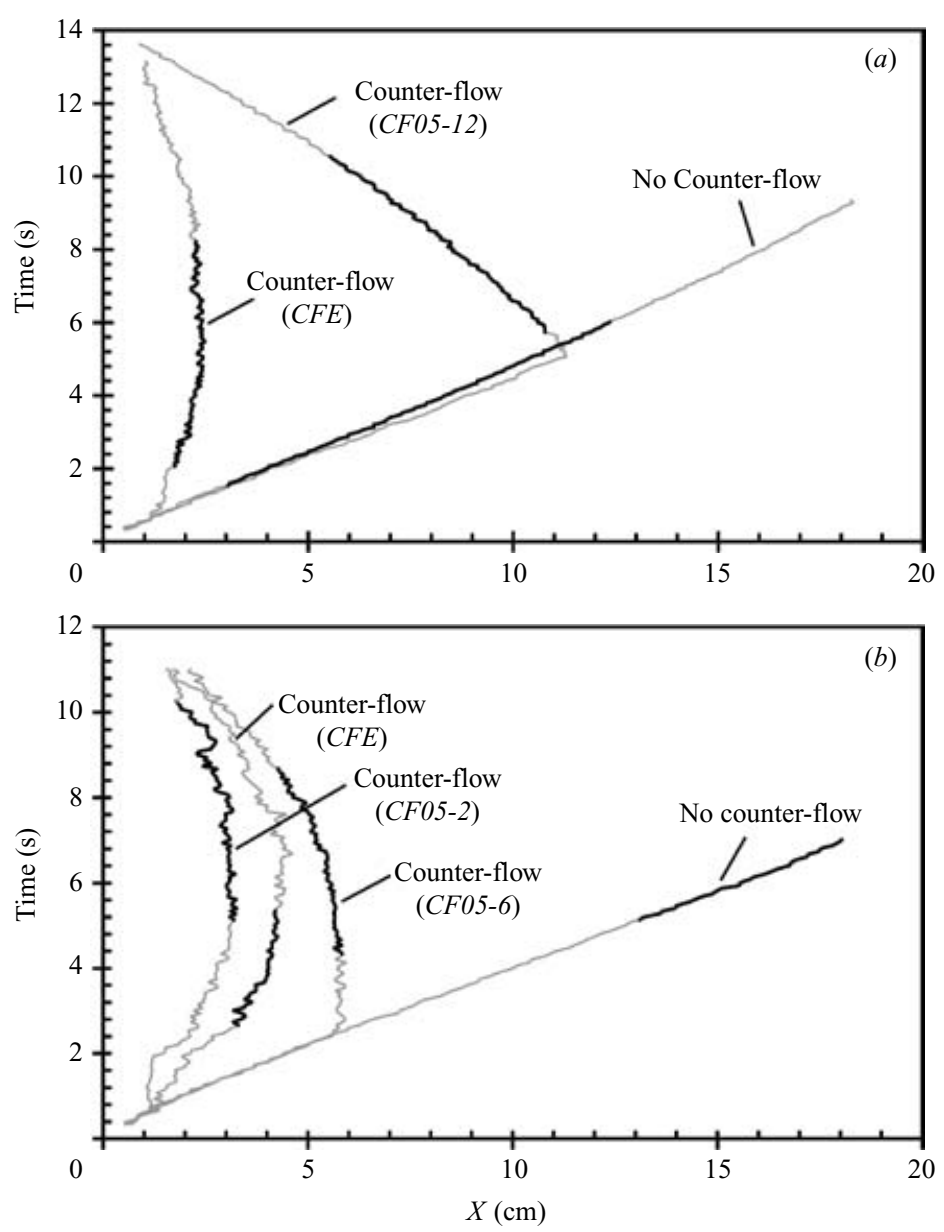

FIGURE 2. Measured vortex ring trajectory for each counter-flow protocol. (a) $L / D=2 ;$ (b) $L / D=4$.

used in the following sections. The basic format of the notation is [vortex generator L/D]-[counter-flow speed]-[initiation delay].

\section{Results}

\subsection{Vortex ring trajectories}

Figure 2 plots the trajectories of vortex rings generated using the protocols in table 1, as measured from the location of peak vorticity in the ring. In the absence of counterflow, cases $L D 2-C F O$ and $L D 4-C F 0$ exhibit the expected ring propagation axially away from the vortex generator under self-induced velocity. The additional shear layer strength and concomitant circulation increase generated in cases $L D 2-C F E$ and $L D 4-C F E$ (i.e. due to counter-flow initiation prior to vortex ring pinch-off) enables these vortex rings to emerge from the vortex generator, despite the presence of counterflow equal to $\left.U_{v}\right|_{C F 0}$. The increased circulation in the $L D 4-C F E$ case is greater than that in the LD4-CF05-2 protocol, leading to the observed larger axial translation away from the vortex generator. Vortex rings generated under the protocols with larger counter-flow initiation delays (i.e. LD2-CF05-12 and LD4-CF05-6) exhibited 

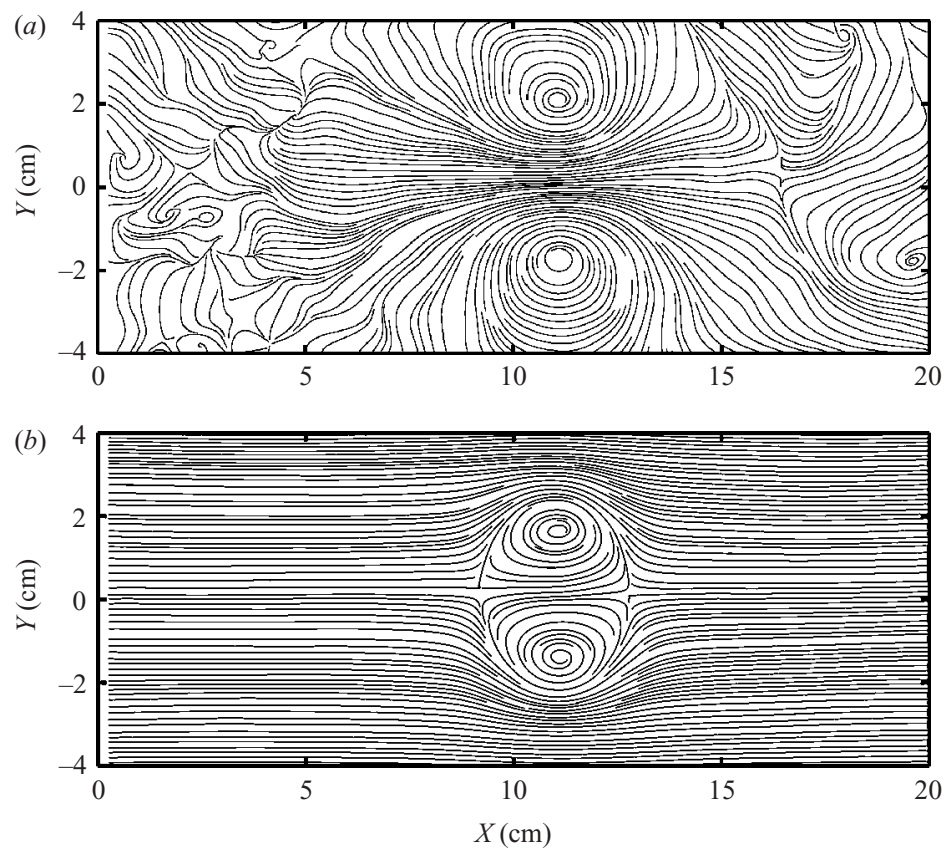

FIGURE 3. Instantaneous streamlines for protocol $L D 2-C F 0$ at time $T=5.34 \mathrm{~s}$.

(a) Laboratory frame; $(b)$ vortex ring frame. Flow is from left to right.

abrupt changes in vortex ring trajectory due to the sudden application of a relatively large counter-flow.

In each of the counter-flow protocols, a trend can be observed at later times in which the counter-flow dominates the self-induced velocity of the rings, convecting the vortex ring structure back toward the vortex generator. This effect can be attributed to the increasing mass of fluid in the vortex bubble as the entrainment process proceeds, slowing and ultimately reversing the forward progress of the bubble.

The darkened portion of each vortex ring trajectory in figure 2 will be the focus of the following investigations. In these regions the vortex ring can be considered isolated, and the transients associated with the formation process (e.g. fluid convection from the vortex generator) have ceased.

\subsection{Fluid entrainment and vorticity distribution}

Measurements of transient fluid entrainment and vorticity distribution in isolated vortex rings were made using DPIV. The primary difficulty associated with such measurements - definition of the vortex boundaries - was overcome by making use of instantaneous streamlines of the flow determined from DPIV velocity field results and a post-processing algorithm created for this purpose.

An example of the instantaneous streamlines measured from a steadily translating vortex ring (protocol $L D 2-C F 0$, time $T=5.34 \mathrm{~s}$ ) is shown in figure $3(a)$, in a laboratory reference frame. The physical extent of the vortex in not evident until the measurement is taken in a frame moving with the ring. This was accomplished by superimposing a free-stream axial flow with magnitude equal to the measured ring axial velocity. Figure $3(b)$ shows the same vortex ring in its moving frame. Its physical extent is well-defined. 
The ring velocity was measured from the axial location of peak vorticity in the cores. A more correct measurement should use an ad hoc vorticity centroid location, such as that suggested by Saffman (1970). However, the difference between Saffman's vorticity centroid location and the vorticity peak appears to remain within experimental error.

The volume of the vortex bubble $\Omega_{B}(t)$ was computed using an ellipsoidal fit based on the measured locations of the front and rear stagnation points as well as the radial extent of the ring. In the event that the rear stagnation point was obscured (e.g. when in close proximity to the vortex generator), this point was defined as the mirror image of the front stagnation point relative to a plane containing the vortex core centres and oriented normal to the axial direction.

Given the measured volume of the vortex bubble $\Omega_{B}(t)$ and the fluid volume $\Omega_{J}(t)$ supplied by the vortex generator,

$$
\Omega_{J}(t)=\frac{\pi D^{2}}{4} \int_{0}^{t} U_{p}(\tau) \mathrm{d} \tau,
$$

the magnitude of entrainment was quantified by the entrained fluid fraction $\eta(t)$, where

$$
\eta(t)=\frac{\Omega_{B}(t)-\Omega_{J}(t)}{\Omega_{B}(t)}=1-\frac{\Omega_{J}(t)}{\Omega_{B}(t)} .
$$

Measurements possess an uncertainty of $8 \%$ to $10 \%$. The primary source of error lies in the measured instantaneous vortex ring velocity that is used to visualize the streamlines in the moving ring frame. This velocity measurement has a first-order effect on the observed location of the ring stagnation points when the free-stream velocity is superimposed.

Figure 4 plots the entrained fluid fraction $\eta(t)$ measured during the darkened portion of each vortex ring trajectory in figure 2 . The trends are generally monotonic in favour of increasing bubble volume. Oscillations in the measured data for protocol LD4-CF05-2 were suspected to be due to rotation of its non-circular vortex cores, which tended to distort the measurement of the radial vortex ring extent. This was confirmed by examination of the vorticity contours, which indicated that the frequency of measurement oscillation exactly matched the frequency of the non-circular core rotation.

It is immediately evident that an extrapolation of the entrainment data to time $T=$ 0 will not lead to the expected condition of zero entrainment fraction at the start of vortex ring formation. This is because convective fluid entrainment is the dominant mechanism at early times, as the generated vortex sheet involutes and captures a substantial portion of ambient fluid near the exit plane of the vortex generator. This captured fluid persists in the vortex bubble indefinitely. The data collected in figure 4 only capture the integrated effect of this early entrainment phase, essentially as an initial condition for the diffusive fluid entrainment of the isolated vortex rings studied here. The dynamics of the convective entrainment process during vortex ring formation are beyond the scope of this paper, although it is interesting to observe in passing that the counter-flow appears to also have a measurable effect on early convective entrainment at the stroke ratio $L / D=4$.

In the absence of counter-flow, the magnitude of fluid entrainment at both piston stroke-to-diameter ratios $L / D=2$ and $L / D=4$ was found to lie between $30 \%$ and $40 \%$ of the bubble volume. These values are higher than those predicted based on a slug model analysis similar to that of Baird et al. (1977). The discrepancy arises due to overestimation of the vortex ring velocity by a boundary-layer-corrected slug 

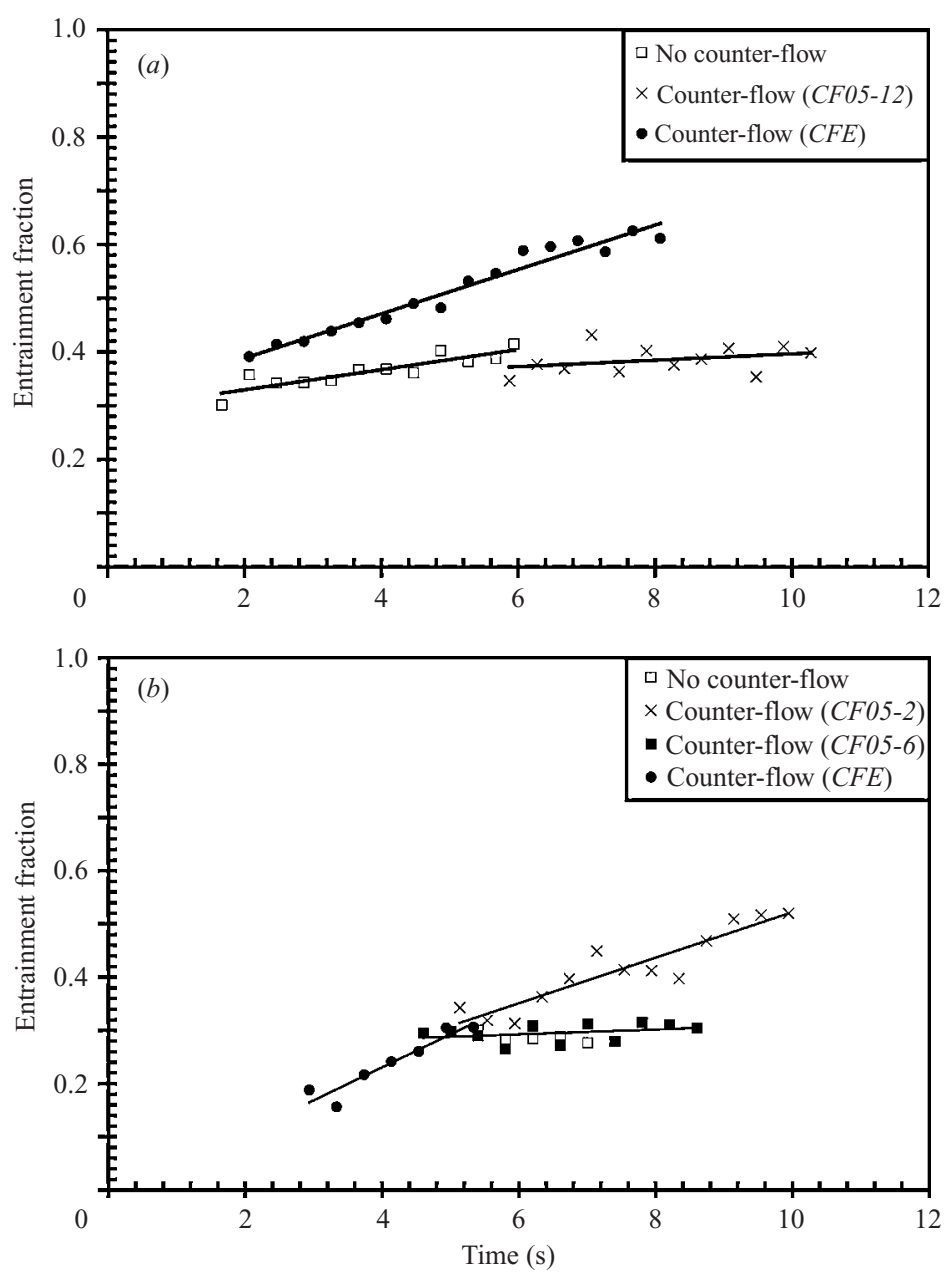

FIGURE 4. Measured entrainment fraction $\eta(t)$ for each counter-flow protocol. (a) $L / D=2 ;(b) L / D=4$.

model (e.g. Shusser et al. 2002; Dabiri \& Gharib 2004a), leading to underestimation of the bubble mass that must share the conserved impulse. The experimental result by Müller \& Didden (1980) of a 40\% entrained fluid fraction lies within the range observed here. A time-dependent trend in the fluid entrainment is not reported by Baird et al. (1977) or Müller \& Didden (1980), limiting any further comparison.

Imposition of a counter-flow was demonstrated to substantially affect fluid entrainment for several of the protocols. An entrainment fraction of nearly $65 \%$ was observed for the $L D 2-C F E$ case, nearly $200 \%$ of the entrainment level for vortex rings generated in the absence of counter-flow. Examination of the vorticity distribution generated by each of the protocols provides the insight necessary to explain the mechanism whereby the process of fluid entrainment is being manipulated. This will be demonstrated in the following development.

An important parameter in the entrainment mechanism described by Maxworthy (1972) is the spatial distribution of vorticity relative to the translating vortex bubble. It is vorticity that has diffused outside the bubble that will reduce the total pressure of ambient fluid near the ring (i.e. by viscous dissipation) to an extent that entrainment 

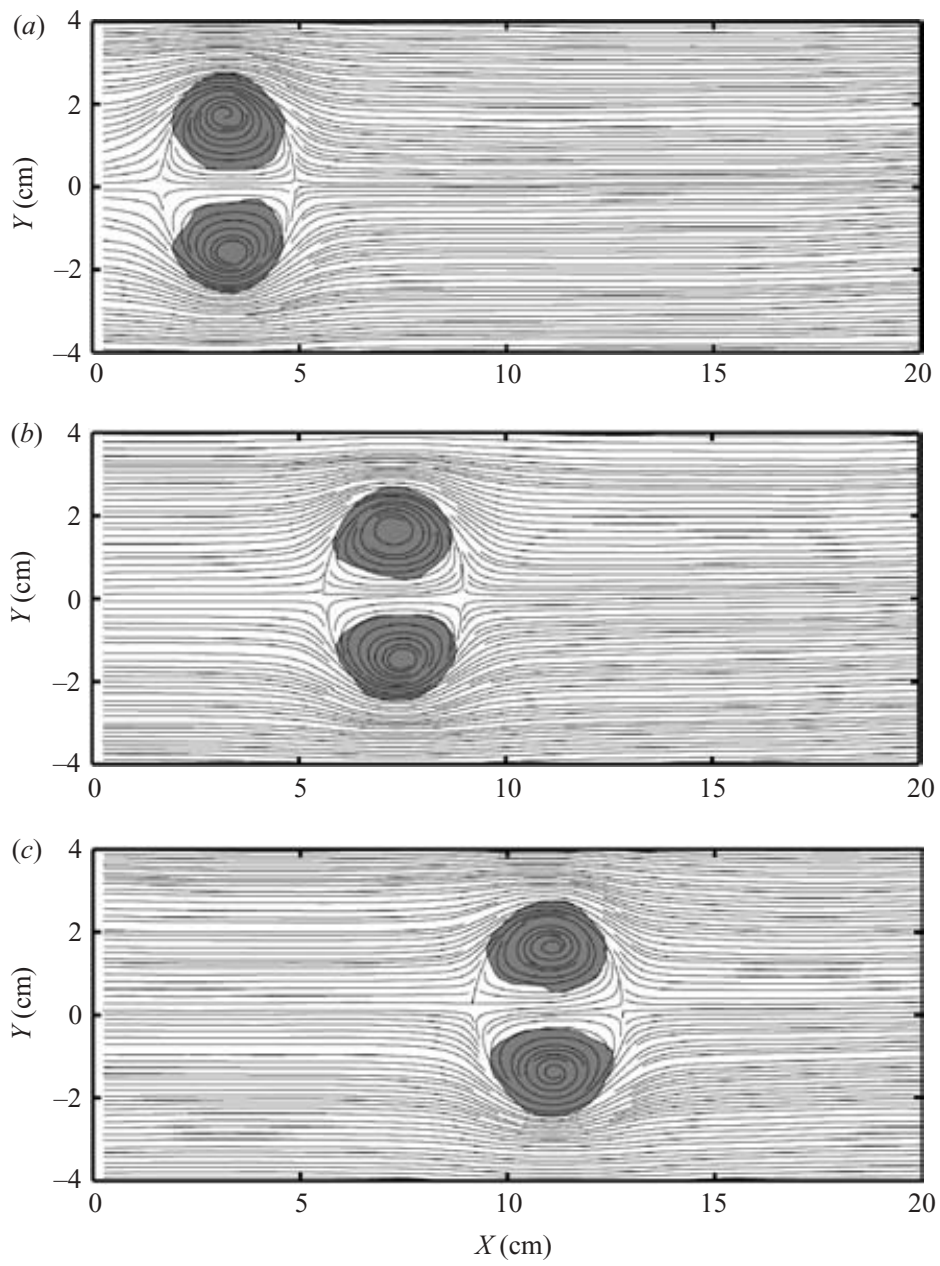

FIGURE 5. Instantaneous streamlines and vorticity patches for protocol $L D 2-C F 0$. (a) $T=1.67 \mathrm{~s}$; (b) $T=3.54 \mathrm{~s}$; (c) $T=5.34 \mathrm{~s}$. Minimum vorticity level is $1 \mathrm{~s}^{-1}$.

can proceed. This relationship can be visualized quantitatively, by superimposing the measured vorticity field on instantaneous streamlines of the flow in the vortex reference frame. Figure 5 plots these data for the protocol $L D 2-C F 0$ at times $T=$ $1.67 \mathrm{~s}, 3.54 \mathrm{~s}$, and $5.34 \mathrm{~s}$. The grey patches represent regions of vorticity magnitude above $10 \%$ of peak vorticity $\left(10 \mathrm{~s}^{-1}\right)$ in the cores.

Consistent with the model of Maxworthy (1972), the vorticity diffuses beyond the radial extent of the vortex bubble where it will interact with ambient fluid. The thickness of this interacting layer remains steady as the vortex bubble translates, as indicated by the plots at three distinct times during the ring evolution. A similar structure is observed in vortex rings generated by the other protocols. The various counter-flow protocols can be distinguished by observing the evolution of the vorticity profiles in time. Figure 6 shows this evolution for cases $L D 2-C F 0$ and $L D 2-C F E$. The vorticity profile evolution for vortex rings generated in the absence of counter-flow (i.e. $L D 2-C F 0$ ) exhibits broadening of an initially Gaussian vorticity distribution. In the process, the location of peak vorticity remains essentially stationary, with a small movement toward the vortex ring axis of symmetry. Thus, relative to the growing 

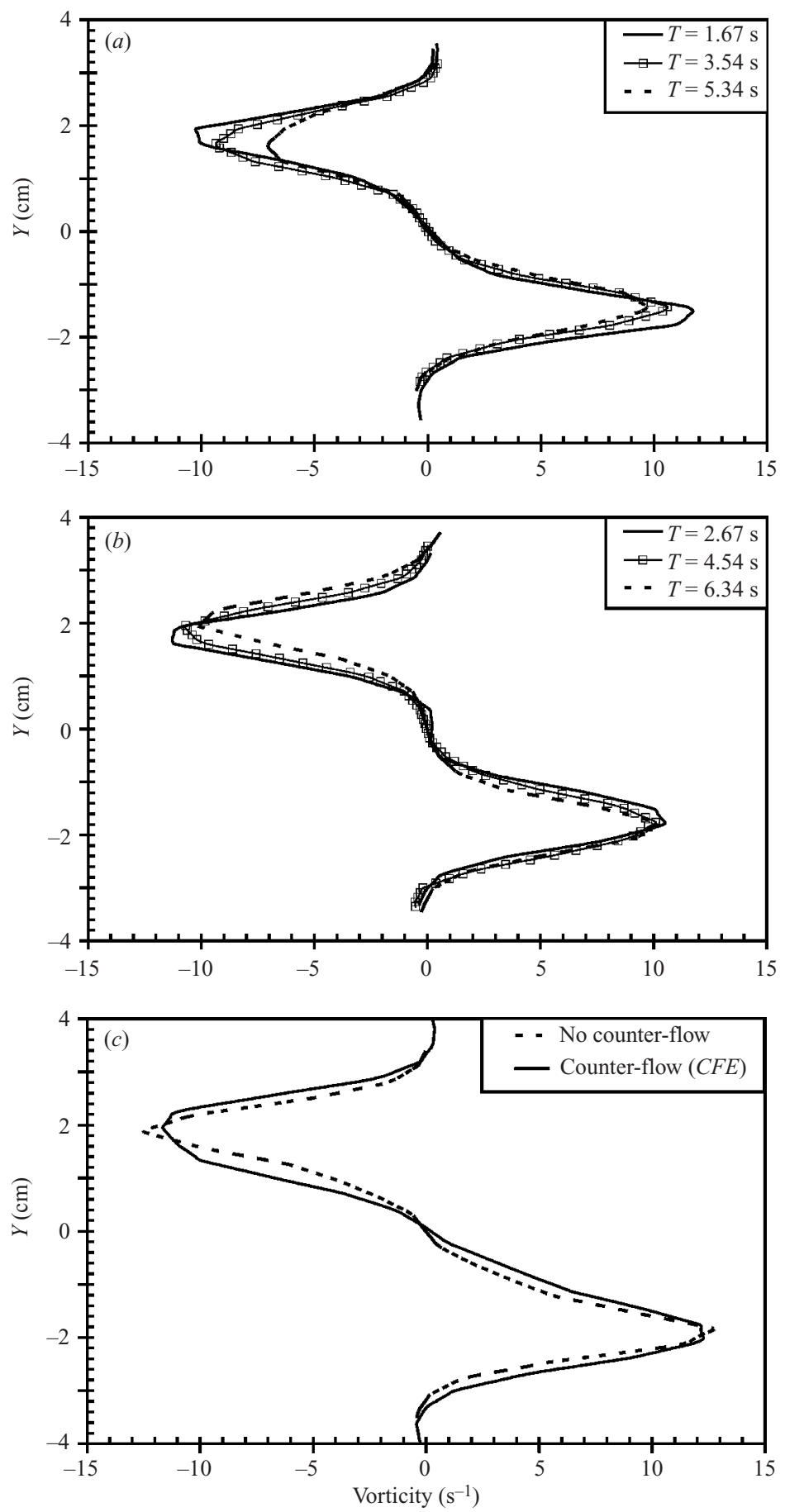

FiguRE 6. $(a, b)$ Temporal evolution of vortex ring vorticity profile for protocols $L D 2-C F 0$ and $L D 2-C F E$, respectively; (c) vorticity profiles for protocols $L D 4-C F 0$ and $L D 4-C F E$ at time $T=2.0 \mathrm{~s}$. 
vortex bubble, the location of peak vorticity is moving away from the ambient fluid on the opposite side of the bounding streamsurface. By contrast, as the vortex rings generated by protocol $L D 2-C F E$ grow, the peak vorticity does not decay as rapidly and its location moves radially away from the vortex ring axis of symmetry (figure $6 b$ ). These effects should enhance the strength of vorticity in the dissipation region, amplifying ambient fluid entrainment. The measurements shown in figure 4 confirm this prediction.

If this physical mechanism is consistent, one can also anticipate that thicker vortex rings - with broader vorticity distribution reaching closer to the bounding streamsurface - will also have enhanced fluid entrainment, relative to a vortex ring with similar aspect ratio but sharper vorticity decay in the radial direction. This, too, is confirmed in the measurements, as indicated by comparison of the vorticity profiles of protocols $L D 4-C F 0$ and $L D 4-C F E$ in figure $6(c)$ and their corresponding entrainment measurements in figure 4.

An additional dynamical process becomes important to the fluid entrainment mechanism for vortex rings generated at piston stroke-to-diameter ratios $L / D$ greater than the formation number $F$. Under these conditions, it has been demonstrated in experiments (Gharib et al. 1998), models (Kelvin 1875; Benjamin 1976; Mohseni \& Gharib 1998; Mohseni 2001), and numerical simulations (Mohseni, Ran \& Colonius 2001) that the vortex ring possesses maximum energy with respect to impulsepreserving rearrangements of the vorticity. The vortex ring cannot accept additional fluid from the vortex generator without violating this energy maximization, leading to the observed pinch-off. Shusser \& Gharib (2000) provide an equivalent statement of this maximization principle, and predict that pinch-off occurs when the velocity of fluid from the vortex generator falls below that of the translating ring. Since the velocity of the ambient fluid is less than that of the vortex generator fluid trailing behind the ring, ambient fluid entrainment must also prohibited after vortex ring pinch-off.

This absence of fluid entrainment is observed for protocols $L D 4-C F 0$ and LD4$C F 05-6$, in which the stroke length-to-diameter ratio of the vortex generator $(L / D=4)$ is greater than the formation number $(F=3.6)$. The entrained fluid fraction is unchanged from its value at the end of the convective entrainment process (i.e. at vortex ring pinch-off). Interestingly, we do observe substantial fluid entrainment for protocols $L D 4-C F 05-2$ and $L D 4-C F E$ well above the formation number $F$, despite the fact that these rings were generated at the same $L / D$ as the non-entraining vortex rings. The apparent contradiction is resolved by the observation that the vortex rings generated by protocols $L D 4-C F 05-2$ and $L D 4-C F E$ do not experience pinch-off. The effect of counter-flow on the shear layer dynamics is to delay vortex ring pinch-off (Dabiri \& Gharib 2004b). Only a minor delay in pinch-off was necessary in the present case, since the stroke length-to-diameter ratio of the vortex generator is only slightly greater than the formation number. However, the delay was sufficient to prevent pinch-off and facilitate fluid entrainment. By contrast, the counter-flow implemented in protocol LD4-CF05-6 was initiated after the formation number, and therefore could not affect the shear layer dynamics or the pinch-off process.

\section{Comparison with Maxworthy (1972)}

The work of Maxworthy (1972) provides the most complete analysis of the diffusive entrainment process in isolated vortex rings that exists in the literature. Due to limitations in the experimental techniques employed therein, many of the model 

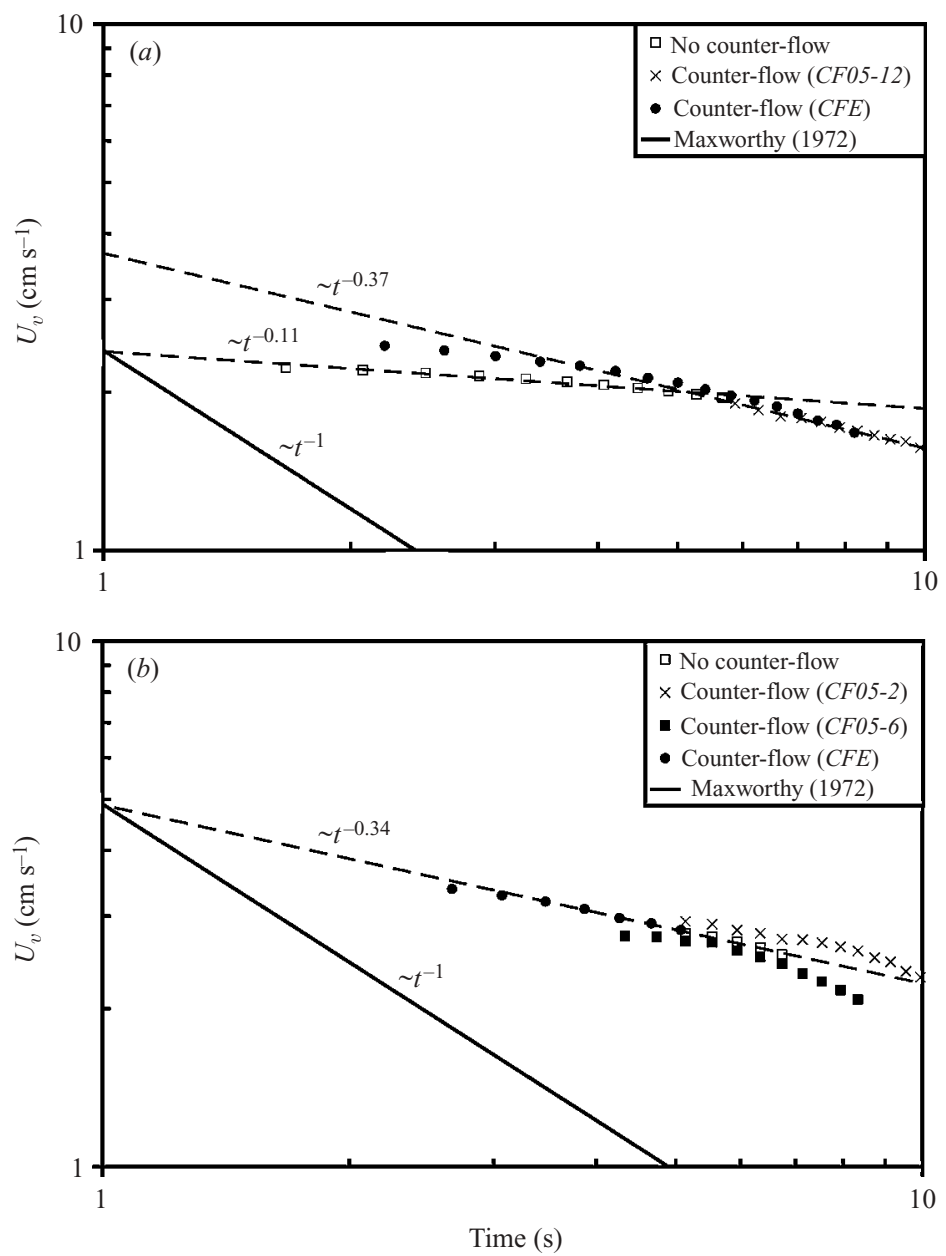

FIGURE 7. Measured vortex ring velocity for each counter-flow protocol. (a) $L / D=2 ;(b) L / D=4$.

predictions could not be validated. Experiments conducted here are sufficient to revisit those analyses and make detailed comparisons. The most important predictions made by Maxworthy (1972) are a -1 power-law decay in the vortex ring propagation velocity $U_{v}$, a $-2 / 3$ power-law decay in vortex ring circulation $\Gamma$, and the formation of a small wake behind the vortex ring. It is prudent to note that the equivalent piston stroke-to-diameter ratio $L / D$ in the experiments of Maxworthy (1972) is not known. However, given that pinch-off is not observed in that study and the Reynolds numbers are in the laminar regime, a comparison with the current results is warranted.

As mentioned in the previous section, the vortex ring velocity in the laboratory frame of reference was measured in these experiments based on the location of peak vorticity in the cores. By subtracting the counter-flow velocity from this measured value, the ring velocity relative to the fluid $U_{v}$ was determined for each of the counterflow protocols. The results are plotted in figure 7. It is evident that the decay rate of the vortex ring velocity in each case is much slower than the -1 power-law observed by Maxworthy (1972). For the majority of protocols, the power-law exponent is closer to $-1 / 3$. Exceptions to this trend occur for the $L D 2-C F 0$ protocol, in which the ring 
decays with a further reduced exponent of approximately $-1 / 9$; and the late-time motion of rings from protocols $L D 4-C F 05-2$ and $L D 4-C F 05-6$, which decay at an increasing rate near the end of the measurements.

Although the present measurements are limited to a single decade of time $\left(10^{0}\right.$ to $10^{1} \mathrm{~s}$ ), this is approximately the same data range reported by Maxworthy (1972). Therefore we can reasonably conclude that the rapid decay in vortex ring velocity reported there is not observed here.

The most likely source of the discrepancy in measured vortex ring velocity lies in the initial conditions of vortex ring generation. Whereas the current experiments utilize a piston-cylinder apparatus to generate the rings, Maxworthy (1972) uses a sharp-edged orifice plate. This can present two prominent effects. First, the magnitude of convective fluid entrainment during vortex ring formation can be substantially altered, leading to different initial conditions for the subsequent diffusive entrainment process than those in the present study. Second, the entrainment of opposite-sign vorticity on the downstream-facing wall of the orifice plate will comprise a larger component than in the present experiments. Maxworthy (1972) predicts the production and convection of this opposite-sign vorticity to affect the dynamics of the evolving vortex rings. It is possible that the translational velocity of the rings is one of the affected parameters, e.g. by vorticity cancellation in the ring, which will reduce its self-induced velocity. The effect of opposite-sign vorticity is further amplified for vortex rings generated at small $L / D$, in which the relative fraction of opposite-sign vorticity increases.

To predict a temporal trend in the vortex bubble circulation, Maxworthy (1972) uses a model that assumes the vortex ring possesses a nominally constant impulse. The model is admittedly limited to large Reynolds numbers and small time scales, so that impulse loss to the wake behind the ring can be neglected. Nevertheless, a $-2 / 3$ power-law decay rate in the ring circulation is predicted. Figure 8 plots the temporal trends in ring circulation for the protocols tested in these experiments. The decay rates are much slower than predicted by Maxworthy (1972). Specifically, the protocols using $L / D=2$ decay with a power-law exponent an order of magnitude smaller than that observed by Maxworthy (1972). The circulation of thicker vortex rings at $L / D=4$ decays slightly faster, but still at a rate much smaller than the $-2 / 3$ power-law.

It is likely that this discrepancy is due to the fact that the present experiments satisfy neither the requirement of large Reynolds number, nor the assumption of short elapsed time. Hence it is also incorrect to assume a constant impulse for the vortex ring.

Using the analytical method of Maxworthy (1972), one can attempt to estimate the decay rate of the ring impulse based on the measured ring velocity and circulation. The impulse $I$ is related to the ring circulation $\Gamma$ and the characteristic dimension of the vortex bubble $\Omega_{B}^{1 / 3}$ by

$$
I=C_{1} \Gamma \Omega_{B}^{2 / 3},
$$

where $C_{1}$ is a dimensionless constant dependent on the bubble shape. The vortex ring velocity $U_{v}$ is related to the ring circulation $\Gamma$ and the characteristic bubble dimension $\Omega_{B}^{1 / 3}$ by

$$
U_{v}=C_{2} \frac{\Gamma}{\Omega_{B}^{1 / 3}},
$$



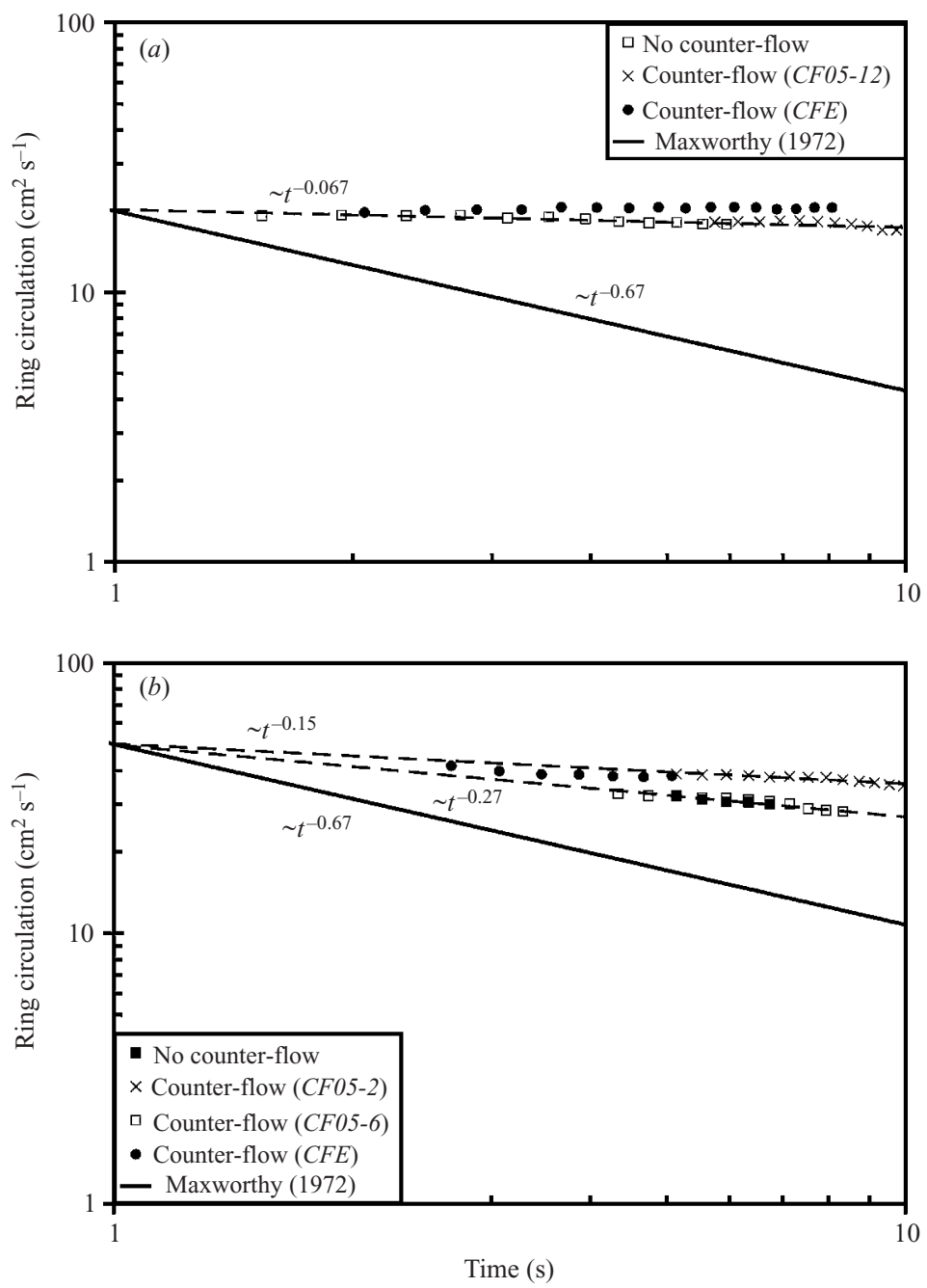

FIGURE 8. Measured vortex ring circulation for each counter-flow protocol. (a) $L / D=2$; (b) $L / D=4$. Note that the data symbol convention in $(b)$ has been altered to avoid data point overlap.

where $C_{2}$ is also a dimensionless constant dependent on the bubble shape. Therefore the ring impulse goes as

$$
I \sim \frac{\Gamma^{3}}{U_{v}^{2}} .
$$

Substituting the measured $-1 / 3$ power-law decay for the vortex ring velocity and $-1 / 10$ power-law decay for the vortex ring circulation (conservatively), the ring impulse is actually predicted to increase with an 11/30 power-law in time! This physically incorrect result of increasing impulse also holds for each of the protocols when the individual velocity and circulation decay rates are input to (4.3) in place of the nominal values above. Therefore we must re-examine the model of Maxworthy (1972). Specifically, the following section will show that that the non-physical result arises due to the assumption of self-similar vortex bubble growth that is implicit 


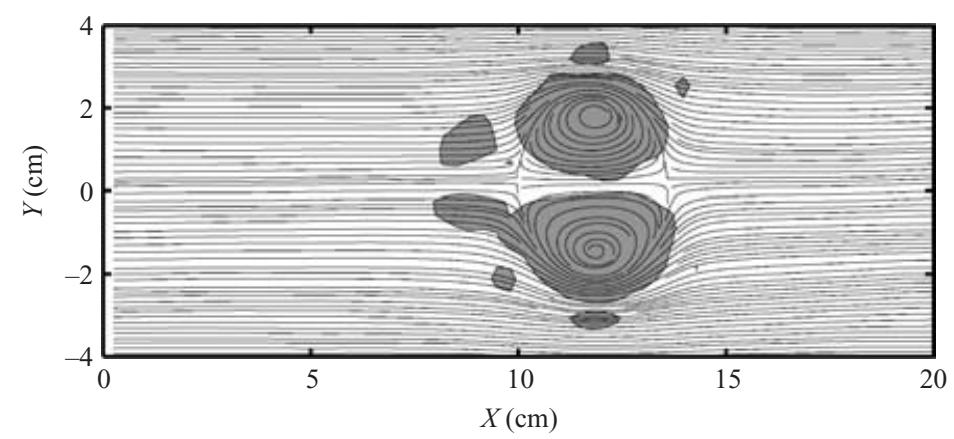

FIGURE 9. Instantaneous streamlines and vorticity patches for protocol $L D 2-C F 0$ at time $T=5.74 \mathrm{~s}$. Minimum vorticity level is $0.3 \mathrm{~s}^{-1}$.

in the use of constant parameters (e.g. $C_{1}$ and $C_{2}$ ) to relate the characteristic ring dimension to the actual bubble shape.

Before concluding this section, it is useful to explore the final important prediction of Maxworthy (1972), namely the formation of a wake behind the vortex ring. Figure 9 plots the vorticity patches and instantaneous streamlines for a vortex ring formed using protocol $L D 2-C F 0$ at time $T=5.74 \mathrm{~s}$. The minimum vorticity level has been reduced to $3 \%$ of the peak vorticity in the vortex core in order to visualize regions of low vorticity. As this represents the upper-bound on the measurement error, the data must be interpreted with caution, especially in regions of the flow with large velocity gradients. Nevertheless, the measurement does appear to capture the presence of symmetric vorticity patches behind the vortex ring, in accordance with the prediction of Maxworthy (1972). The existence of this wake structure emphasizes the fact that the increase in vortex ring impulse predicted by the model above must be incorrect. Given the consistency of the observed wake with the aforementioned physical mechanism for diffusive fluid entrainment proposed by Maxworthy (1972), it will henceforth be assumed correct, and will be used in the following section to derive an improved quantitative model for the process.

\section{A quantitative model for diffusive fluid entrainment}

The physical process of diffusive entrainment is assumed to be essentially that of Maxworthy (1972), as described in the Introduction. Our model will make the corollary assumption that the fraction of ambient fluid flux into the dissipation region that is entrained by the vortex ring is proportional to the fraction of energy lost by the ambient fluid in the same region, i.e.

$$
\frac{\mathrm{d} \Omega_{B} / \mathrm{d} t}{\mathrm{~d} \Omega_{D} / \mathrm{d} t} \sim \frac{\mathrm{d} E_{L} / \mathrm{d} t}{\mathrm{~d} E_{I} / \mathrm{d} t}
$$

where $\mathrm{d} \Omega_{B} / \mathrm{d} t$ is the flux of entrained fluid from the dissipation region into the vortex ring, $\mathrm{d} \Omega_{D} / \mathrm{d} t$ is the total flux of fluid into the dissipation region, $\mathrm{d} E_{L} / \mathrm{d} t$ is the rate of ambient fluid energy loss due to viscous dissipation in the dissipation region, and $\mathrm{d} E_{I} / \mathrm{d} t$ is the rate of ambient fluid energy entering the dissipation region.

The rate of energy loss $\mathrm{d} E_{L} / \mathrm{d} t$ (per unit mass) is computed using the twodimensional incompressible dissipation function $\Phi$ in the energy equation (i.e. the scalar product of the momentum equation with the velocity vector $\boldsymbol{u}$; see Batchelor 
1967):

$$
\frac{\mathrm{d} E_{L}}{\mathrm{~d} t}=\Phi=2 v\left[\left(\frac{\partial u}{\partial x}\right)^{2}+\left(\frac{\partial v}{\partial r}\right)^{2}+\frac{1}{2}\left(\frac{\partial v}{\partial x}+\frac{\partial u}{\partial r}\right)^{2}\right],
$$

where $u$ and $v$ are the velocity components in the axial $x$ and radial $r$ directions, respectively. The magnitude of the dissipation function will be proportional to the kinematic viscosity $v$, and to a characteristic speed $U_{v}$ and length scale $\Omega_{B}^{1 / 3}$ over which changes in fluid velocity occur, i.e.

$$
\frac{\mathrm{d} E_{L}}{\mathrm{~d} t}=\Phi=S_{1} v U_{v}^{2} \Omega_{B}^{-2 / 3} .
$$

In (5.3), $S_{1}$ is a dimensionless shape factor that is dependent on time and other parameters to be delineated shortly. This is distinct from the assumption of constant shape factors used by Maxworthy (1972).

The rate of ambient fluid energy entering the dissipation region (per unit mass) is directly proportional to the square of the fluid velocity and inversely proportional to the time scale over which the process occurs:

$$
\frac{\mathrm{d} E_{I}}{\mathrm{~d} t}=U_{v}^{2} T_{d}^{-1} .
$$

This characteristic dissipation time scale $T_{d}$ can be replaced by the characteristic velocity and bubble dimension as $T_{d}=S_{2} \Omega_{B}^{1 / 3} U_{v}^{-1}$, where $S_{2}$ is a second dimensionless shape factor. With this substitution, (5.4) becomes

$$
\frac{\mathrm{d} E_{I}}{\mathrm{~d} t}=S_{2} U_{v}^{3} \Omega_{B}^{-1 / 3} .
$$

Finally, the volume flux of fluid into the dissipation region is

$$
\frac{\mathrm{d} \Omega_{D}}{\mathrm{~d} t}=S_{3}\left(\nu T_{d}\right)^{1 / 2} \Omega^{1 / 3} U_{v}=S_{2}^{1 / 2} S_{3} v^{1 / 2} \Omega_{B}^{1 / 2} U_{v}^{1 / 2},
$$

where the radial extent of the dissipation region is proportional to $\left(v T_{d}\right)^{1 / 2}$. Substituting (5.3), (5.5), and (5.6) into (5.1) gives an expression for the vortex bubble growth as a function of the characteristic ring velocity and dimension, and the shape factors:

$$
\frac{\mathrm{d} \Omega_{B}}{\mathrm{~d} t}=S_{1} S_{2}^{-1 / 2} S_{3} v^{3 / 2} U_{v}^{-1 / 2} \Omega_{B}^{1 / 6} .
$$

Henceforth, we will consider a dimensionless lumped shape factor $S=S_{1} S_{2}^{-1 / 2} S_{3}$ and unit viscosity. As mentioned above, the shape factor is no longer assumed constant, and is instead dependent on time and the set of parameters that will affect the shape and size of the ring from the start of diffusive entrainment: the instantaneous circulation of the ring $\Gamma(t)$; the duration of vortex generator fluid ejection $T_{e}$; the rate of change of the entrained fluid fraction $\mathrm{d} \eta / \mathrm{d} t$; and the vortex generator exit diameter $D$. Given this functional dependence, the shape factor can be expressed in terms of a single dimensionless parameter,

$$
S=\widehat{S} \frac{\mathrm{d} \eta}{\mathrm{d} t} \Gamma D^{-2} T_{e} t
$$

where $\widehat{S}$ is a dimensionless constant. Substituting for $S$ in (5.7) using this result and rearranging with the use of (3.2), we obtain

$$
\Omega_{B}(t)=\Omega_{B}^{0}+\widetilde{S} \Gamma(t)^{6 / 11} D^{-12 / 11} T_{e}^{6 / 11} \Omega_{J}^{6 / 11} U_{v}(t)^{-3 / 11} t^{6 / 11} .
$$




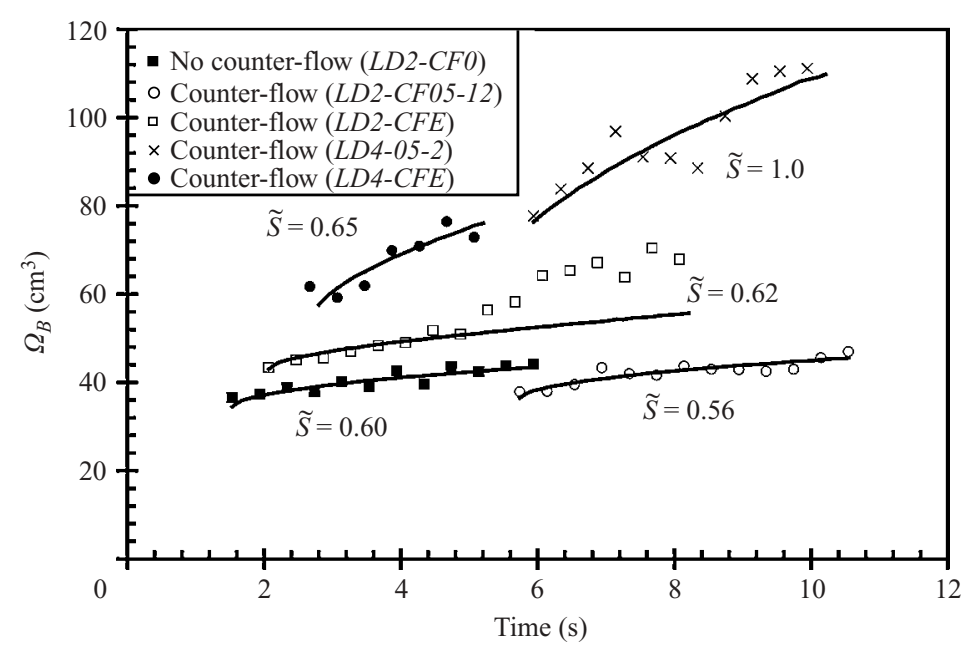

FIGURE 10. Comparison of predicted vortex bubble growth to experimental measurements. Non-entraining protocols (i.e. LD4-CFO and LD4-CF05-6) are excluded.

Note that (5.9) is dimensionally correct with $\widetilde{S}=\widehat{S}^{6 / 11}$ as a dimensionless constant, when the unit viscosity from (5.7) is included. The time variable $t$ in (5.9) is measured relative to the beginning of diffusive entrainment. Therefore the constant $\Omega_{B}^{0}$ is included as the volume of the vortex bubble at the beginning of the diffusive entrainment phase. The magnitude of $\Omega_{B}^{0}$ will be determined by the dynamics of the preceding convective entrainment phase. In the following, $\Omega_{B}^{0}$ will be estimated based on the earliest data points in each measurement series, although it is recognized that each measurement did not necessarily commence immediately after the convective entrainment phase.

The large number of parameters in (5.9) may be reduced via simplifying assumptions regarding the dependence of the vortex ring velocity $U_{v}(t)$ on its circulation (e.g. Norbury 1973), and the dependence of the circulation on the variables $\Omega_{J}, D$, and $T_{e}$ (e.g. slug model approximation, cf. Shariff \& Leonard 1992). However, since the data for every parameter are readily available in the present case, we will not implement those approximations at this time.

Figure 10 plots the predicted vortex bubble growth law in (5.9) for each of the counter-flow protocols (excluding the non-entraining protocols LD4-CFO and LD4CF05-6), along with the measured vortex bubble growth in each case. The shape constant for each curve is indicated in the figure. A consistently high level of agreement is observed in each case, with the exception of the late-time behaviour of protocol $L D 2-C F E$. This discrepancy is probably due to the close proximity of the ring to the vortex generator, as indicated by its trajectory in figure 2 . It appears the vortex generator may have prevented normal deposition of ring circulation into a wake, allowing the ring to achieve substantially enhanced entrainment at later times when the ring circulation is normally decaying. This explanation is supported by the observed lack of ring circulation decay for protocol $L D 2-C F E$ in figure 8. Although this result emphasizes the role of vorticity distribution in the entrainment process, these modified entrainment dynamics cannot be captured by the current model.

The dimensionless shape constant $\widetilde{S}$ is of order unity and exhibits only modest variation across the range of experimental results. It appears that the constant scales 
with increasing entrainment enhancement, but the current data are insufficient to draw firm conclusions.

\section{Conclusions}

Novel experimental techniques have been developed to probe the dynamics of isolated vortex rings. The counter-flow protocols have enabled empirical study of vortex ring evolution in a moving frame of reference, effectively eliminating many of the common obstacles to progress in previous efforts. Quantitative velocimetry has elucidated the structure of the rings, and a diverse set of experimental conditions has indicated the special role of vorticity distribution and vortex ring pinch-off in the diffusive process of ambient fluid entrainment. In a normal diffusive process (i.e. without counter-flow enhancement), entrained fluid fractions between $30 \%$ and $40 \%$ were measured. Various counter-flow protocols demonstrated the possibility of substantial entrainment augmentation, up to a $65 \%$ entrained fluid fraction in these experiments.

Wake formation behind vortex rings during the entrainment process was observed in these experiments, confirming the physical model for diffusive entrainment suggested by Maxworthy (1972). The corresponding measurements of ring velocity and circulation showed substantial discrepancy, however. Differences in observed ring velocity are probably due to dissimilar boundary conditions in the vortex generator of the previous experiments and the current study, while the overestimate in predicted circulation decay can be attributed to the invalid assumptions of constant ring impulse and self-similar growth in the previous analysis. The present results should serve to caution efforts to describe vortex ring dynamics using constant-impulse or constant-volume assumptions. Although such approximations may be reliable early in the diffusive stage of fluid entrainment, they are inappropriate during both ring formation and late-time ring propagation.

A model for diffusive fluid entrainment has been developed here that does not assume a self-similar shape for vortex rings as they evolve, nor does it require a nominally constant ring impulse. Dimensional analysis and physical arguments were used to derive the growth rate of the vortex bubble as a function of ring circulation and propagation speed. Predictions of the model were found to agree well with measurements of the normal diffusive entrainment process, and it is hypothesized that the shape constant $\widetilde{S}$ may scale with increasing entrainment enhancement. Unfortunately, the discovery that the vortex rings do not evolve self-similarly limits our ability to predict trends in the ring impulse using formulae from thin-core approximations or steady solution families (e.g. Fraenkel 1972; Norbury 1973).

Future efforts must be directed toward achieving a better understanding of the rapid convective entrainment process that occurs during vortex ring formation. One can infer from the present experiments that this early phase of entrainment will be responsible for much of the entrained fluid fraction that persists in the vortex rings. The current experimental method was not sufficiently accurate to resolve that convective entrainment process, but it is possible that a refinement of the technique introduced here might be effective. Despite the absence of dynamical information regarding the convective fluid entrainment process, the present experiments have definitively shown a substantial contribution of ambient fluid entrainment to the dynamics of vortex rings. As improved ideal vortex ring models are being developed, it will be prudent to account for this phenomenon. 
These results may have important implications for pulsed jet systems that possess a capability to manipulate the vortex ring velocity and/or vorticity distribution. Notwithstanding the many manmade applications that may be designed to exploit these parameters in the future, various animals may have already achieved success in this endeavour. For example, it is known that many pulsed-jet animal swimmers that are dependent on self-generated vortical flows for feeding are narrowly tuned for a specific swimming speed regime. Since the relative speed of vortex rings generated during swimming is coupled to the prevalent swimming speed regime of the animal, it will be interesting to further examine the relationship between swimming regime and the effectiveness of entrainment mechanisms in these animals.

The authors gratefully acknowledge P. S. Krueger for helpful discussions and design of the original coaxial flow apparatus that was modified for these experiments. The reviewers of this paper are also appreciated for their useful comments and suggestions. This work has been conducted with the support of National Science Foundation Grant 0309671.

\section{REFERENCES}

Baird, M. H. I., Wairegi, T. \& Loo, H. J. 1977 Velocity and momentum of vortex rings in relation to formation parameters. Can. J. Chem. Engng 55, 19-26.

Batchelor, G. K. 1977 An Introduction to Fluid Dynamics. Cambridge University Press.

BENJAMIN, T. B. 1976 The alliance of practical and analytical insights into the non-linear problems of fluid mechanics. In Applications of Methods of Functional Analysis to Problems in Mechanics (ed. P. Germain \& B. Nayroles). Lecture Notes in Mathematics, vol. 503, pp. 8-28. Springer.

DABIRI, J. O. \& Gharib, M. 2004a A revised slug model boundary layer correction for starting jet vorticity flux. Theor. Comput. Fluid Dyn. 17, 224-226.

DABIRI, J. O. \& GHARIB, M. $2004 b$ Delay of vortex ring pinch-off by an imposed bulk counter-flow. Phys. Fluids 16, L28-L30.

Fraenkel, L. E. 1972 Examples of steady vortex rings of small cross-section in an ideal fluid. J. Fluid Mech. 51, 119-135.

Gharib, M., Rambod, E. \& Shariff, K. 1998 A universal time scale for vortex ring formation. J. Fluid Mech. 360, 121-140.

Kelvin, LoRD 1875 Vortex statics. Collected Works vol. 4, pp. 115-128. Cambridge University Press.

Krueger, P. S., DABiri, J. O. \& Gharib, M. 2003 The formation number of vortex rings formed in the presence of uniform background co-flow. Phys. Fluids 15, L49-L52.

Liess, C. \& DidDen, N. 1976 Experimente zum Einfluss der Anfangsbedingungen auf die Instabilität von Ringwirblen. Z. Angew. Math. Mech. 56, T206-T208.

Linden, P. F. \& TuRner, J. S. 2001 The formation of 'optimal' vortex rings, and the efficiency of propulsion devices. J. Fluid Mech. 427, 61-72.

Maxworthy, T. 1972 The structure and stability of vortex rings. J. Fluid Mech. 51, 15-32.

Maxworthy, T. 1977 Some experimental studies of vortex rings. J. Fluid Mech. 80, 465-495.

MoHSEnI, K. 2001 Statistical equilibrium theory for axisymmetric flows: Kelvin's variational principle and an explanation for the vortex ring pinch-off process. Phys. Fluids 13, 1924-1931.

Mohseni, K. \& Gharib, M. 1998 A model for universal time scale of vortex ring formation. Phys. Fluids 10, 2436-2438.

Mohseni, K., Ran, H. Y. \& Colonius, T. 2001 Numerical experiments on vortex ring formation. J. Fluid Mech. 430, 267-282.

MüLler, E. A. \& DidDEN, N. 1980 Zur erzeugung der zirkulation bei der bildung eines ringwirbels an einer dusenmundung. Stroj. Casop. 31, 363-372.

Norbury, J. 1973 A family of steady vortex rings. J. Fluid Mech. 57, 417-431.

Prandtl, L. \& Tietjens, O. G. 1934 Fundamentals of Hydro and Aeromechanics. Dover.

REYNOLDS, O. 1876 On the resistance encountered by vortex rings, and the relation between vortex rings and the streamlines of a disk. Nature 14, 477. 
Saffman, P. G. 1970 The velocity of viscous vortex rings. Stud. Appl. Maths 49, 371-380.

Shariff, K. \& LeOnard, A. 1992 Vortex rings. Annu. Rev. Fluid Mech. 24, 235-279.

Shusser, M. \& Gharib, M. 2000 Energy and velocity of a forming vortex ring. Phys. Fluids 12, 618-621.

Shusser, M., Gharib, M., Rosenfeld, M. \& Mohseni, K. 2002 On the effect of pipe boundary layer growth on the formation of a laminar vortex ring generated by a piston/cylinder arrangement. Theor. Comput. Fluid Dyn. 15, 303-316.

Widnall, S. E. \& Sullivan, J. P. 1973 On the stability of vortex rings. Proc. R. Soc. Lond. A 332, 335-353.

Willert, C. E. \& Gharib, M. 1991 Digital particle image velocimetry. Exps. Fluids 10, 181-193. 